\title{
Is the largest aqueous gold cluster a superatom complex? Electronic structure \& optical response of the structurally determined $A u_{146}(\mathrm{pMBA})_{57}$.
}

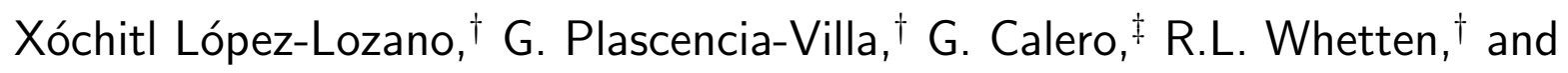 \\ H.-Ch. Weissker*, ${ }^{*}, \S$ \\ $\dagger$ Department of Physics 83 Astronomy, The University of Texas at San Antonio, One \\ UTSA circle, 78249-0697 San Antonio, TX., USA \\ $\ddagger D$ pt. of Structural Biology, University of Pittsburg, Pittsburg, PA, USA. \\ \Aix Marseille University, CNRS, CINaM UMR 7325, 13288, Marseille, France \\ $\S$ European Theoretical Spectroscopy Facility \\ E-mail: weissker@cinam.univ-mrs.fr
}




\begin{abstract}
The new water-soluble gold cluster $\mathrm{Au}_{146}(\mathrm{pMBA})_{57}$, the structure of which has been recently determined at sub-atomic resolution by Vergara et al., 1 is the largest aqueous gold cluster ever structurally determined and likewise the smallest cluster with a stacking fault. The core presents a twinned truncated octahedron, while additional peripheral gold atoms follow a $\mathrm{C}_{2}$ rotational symmetry. According to the usual counting rules of the superatom complex (SAC) model, the compound attains a number of $92 \mathrm{SAC}$ electrons if the overall net charge is 3- (three additional electrons). As this is the number of electrons required for a major shell closing, the question arises if $\mathrm{Au}_{146}(\mathrm{pMBA})_{57}$ should be regarded as a superatom complex. Starting from the experimental coordinates we have analyzed the structure using density-functional theory. The optimized (relaxed) structure retains all the connectivity of the experimental coordinates, while removing much of its irregularities in interatomic distances, thereby enhancing the $\mathrm{C} 2$ symmetry feature. Analyzing the angular-momentum projected states, we show that, despite a small gap, the electronic structure does not exhibit SAC model character. In addition, optical absorption spectra are found to be relatively smooth compared to the example of the $\mathrm{Au}_{144}(\mathrm{SR})_{60}$ cluster. The $\mathrm{Au}_{146}(\mathrm{SR})_{57}$ cluster does not derive its stability from SAC character; it cannot be considered a superatom complex.
\end{abstract}

\title{
Introduction
}

Monolayer-protected gold clusters have been commercially available since many years ("PeptideGold"2 ${ }^{2}$, and they are used in a multitude of biological and medical investigations over the past decade which all require aqueous-phase compatibility; applications include bioconjugation chemistry,,$^{3}$ protein tagging, ${ }^{2}$ biomolecule labeling, ${ }^{4}$ inhibition of HIV fusion,, 5 as well as growth inhibition of bacteria. ${ }^{6}$

But their approved use is severely inhibited by a lack of precise knowledge of their structure, composition, and related fundamental properties. Herein, we present a comprehen- 
sive study of the electronic and optical properties of the novel water-soluble gold cluster $\mathrm{Au}_{146}(\mathrm{pMBA})_{57}$, the structure of which has been recently determined at sub-atomic resolution by Vergara et al. 1

The stability and chemical nature of many metal clusters has been found to follow the super-atom model, ${ }^{7}$ in which the delocalized states (derived from the $N s$ electrons in noble metals, where $N$ is the number of noble metal atoms) belong to distinct values of angular momentum and follow an Aufbauprinzip similar to that found for the spherical jellium model. .8 In medium-sized gold clusters, the $n^{*}$ delocalized orbitals derive mainly from atomic 6s orbitals. The Aufbau rule is ${ }^{9}\left|1 S^{2}\right| 1 P^{6}\left|1 D^{10}\right| 2 S^{2} 1 F^{14}\left|2 P^{6} 1 G^{18}\right| 2 D^{10} 3 S^{2} 1 H^{22} \mid \ldots$, leading to shell closures at $n^{*}=2,8,18,34,58,92,138 \ldots$ which explain particular stability when the electron count matches one of these "magic numbers."

This observation was generalized as "super-atom complex model" (SAC model) $\underline{910}$ to describe monolayer-protected clusters of roughly globular shape. It assumes that stabilizing ligands may either withdraw electrons or localize electrons into covalent bonds. Assuming the withdrawal of one electron for each of the $M$ pseudohalide ligands and noting that each of the $\mathrm{N}$ gold atoms provides one $6 \mathrm{~s}$ electron, one obtains the requirement that $n^{*}=N-M-z$ be equal to one of the magic numbers listed above for an electronically closed superatom complex, where $\mathrm{z}$ is the overall charge. ${ }^{9}$ If electronic shell structure is an important factor in the stability of the compound, there should be a clear gap after the shell closure, which implies a deep minimum in the electronic density of states. ${ }^{9}$ Projection of the Kohn-Sham orbitals onto spherical harmonics allows for the decomposition of the electronic density of states, $\stackrel{9111}{ }$ clearly showing the SAC character of many clusters.

The number of clusters that have been synthesized, purified, and studied has increased immensely over the last few years. $\frac{12}{12}$ Among the monolayer-covered Au clusters, the size range of approximately 140 to 150 gold atoms is of great interest, with the particularly stable, ubiquitous structure $\mathrm{Au}_{144}(\mathrm{SR})_{60}$ playing a special role for multiple reasons: 
(i) Its size is such that the localized surface-plasmon resonance (LSPR) is not yet visible, although an incipient LSPR has been discussed.13.14

(ii) The cluster has been shown in 2014 to exhibit a multitude of discrete peaks in the absorption spectra which reflect the discrete electronic structure. 15

(iii) Various groups have synthesized and studied the compound, with different thiolate ligand rest groups, demonstrating a high degree of reproducibility (see Ref. 16 for a collection).

(iv) Different $\mathrm{Au}_{144}$-based alloy clusters have been studied both experimentally and theoretically. In particular, the general effect of alloying with silver was studied, $17-20$ as well as the interesting case of adding copper which seems to produce a plasmon-like resonance, 2122 the origins of which remain, however, unclear. $\underline{23}$

(v) Moreover, the size of 144 gold atoms is where fcc-based (i.e., bulk-like or similar) structures start to dominate,,$\frac{24}{2}$ whereas for smaller clusters molecule-like structures prevail. Specifically for the size of 144 gold atoms and pMBA ligands, polymorphism has recently been inferred: a truncated octahedron structure may coexist with the well-known icosahedral structure. 25

(vi) Finally, it has been shown that the electronic structure around the HOMO-LUMO gap of $\mathrm{Au}_{144}(\mathrm{SR})_{60}$ corresponds to super-atom-like states. 26

The new monolayer-protected gold cluster $\mathrm{Au}_{146}(\mathrm{pMBA})_{57}$, the structure of which has been recently determined by Vergara et al., 1 is the largest aqueous gold cluster ever structurally determined and likewise the smallest cluster with a stacking fault. The core presents a twinned truncated octahedron, while additional peripheral gold atoms follow a $\mathrm{C}_{2}$ rotational symmetry. With the usual electron counting rules as mentioned above, ${ }^{9}$ a charge state of 3- (three additional electrons) leads to a number of SAC electrons of $146-57-(-3)=92$, which would correspond to a SAC shell closing. This raises the question if such a shell closing could explain the stability of the cluster compound.

In the present manuscript, we use density-functional theory (DFT) calculations to show 
that despite the proximity of the magic SAC number of 92 electrons, the $\mathrm{Au}_{146}(\mathrm{pMBA})_{57}$ cluster does not derive its sstability from super-atom complex character. After a description of the technical details, we discuss briefly the types of bonds and the bond lengths in the relaxed structure. Subsequently, we analyze the electronic structure and the optical response of the cluster and show that the nature of a superatom complex is not realized.

\section{Technical details}

We start from the experimental structure of $\mathrm{Au}_{146}(\mathrm{pMBA})_{57}$ determined as reported by Vergara et $a l .1]$ In order to obtain a tractable size for the ab initio calculations, we have removed the restgroup $\mathrm{R}$ of all the p-MBA ligands, leaving only the carbon atoms directly bound to the sulfur atoms in place. Subsequently, the structure was saturated by hydrogen atoms (resulting in $\mathrm{R}=\mathrm{CH}_{3}$ methyl groups) in such a way that one of the hydrogen atoms pointed to the midpoint of the line connecting the two adjacent Au atoms (i.e., the plane defined by S-C-H intersecting this line at its midpoint). For comparison, we have also studied an isoelectronic-substitution model where each complete ligand molecule (pMBA) is replaced by one $\mathrm{Cl}$ atom.

The structure was then relaxed using the VASP code $27 \sqrt[29]{29}$ using density-functional theory (DFT) with the projector-augmented wave method (PAW) ${ }^{29}$ and employing the LDA exchange-correlation functional and, for comparison, the GGA functional PBE,, 30 until all forces were smaller than $0.06 \mathrm{eV} / \AA$. A charge state of 3 - (three additional electrons) was used to comply with the expected electronic shell closing at 2350 electrons according to the SAC model. Relaxation was slow, it appears clear that the total energy surface is composed of many shallow local minima (in particular, sensitive to the orientation of the methyl-group ligands). The chlorine-substituted model relaxed much faster to very small forces (smaller than $0.001 \mathrm{eV} / \AA$ ). The energy difference between the initial and the relaxed structure is about $17 \mathrm{eV}$ for the relaxation using the LDA functional. 
The optical absorption spectra are calculated by TDDFT using the real-space code octopus $^{3132}$ with the time-evolution formalism ${ }^{33}$ and the PBE exchange-correlation potential ${ }^{30}$ for coherence with previous studies. ${ }^{15116}$ A comparison of LDA and PBE spectra for identical geometries is shown in Fig. S4 the ESI. Norm-conserving Troullier-Martins pseudopotentials ${ }^{34}$ have been used which, similarly as in our previous work, $\frac{15116}{1 n c l u d e}$ the $d$ electrons in the valence (11 valence electrons for each Au atom, i.e., in total 2,350 electrons for the $\mathrm{R}=\mathrm{CH}_{3}$ calculations). The spacing of the real-space grid was set to $0.20 \AA$, the radius of the spheres centered around each atom which make up the calculation domain to $5 \AA$.

The total energy is used to monitor the stability of the propagation. The time step for the propagation was set to $0.00197 \mathrm{fs}$, the propagation time was $25 \mathrm{fs}$, and the propagation was carried out by means of the Approximated Enforced Time-Reversal Symmetry (AETRS) propagator $^{35}$ as implemented in the octopus code. The 25 -fs period provides a transformlimited resolution of $40 \mathrm{THz}$, or $\sim 0.16 \mathrm{eV}$, for all the spectra computed and shown in the Figures reported herein.

The results of the time-evolution formalism are equivalent to those obtained using the transition-based Casida formalism. $\frac{3637}{}$ A comparison of the time-evolution results with a transition-based calculation is shown in the Supplementary Material of Ref. 15 for the thiolate-ligand covered $\mathrm{Au}_{38}$ cluster. For a bare Au cluster, a similar comparison is shown in the supplementary material of Ref. 38. The good agreement shows likewise that the technical parameters of our calculations are well controlled.

The electronic energies shown herein are from the VASP calculation. The plotted wave functions are from an octopus calculation. In order to obtain the angular-momentumprojected density of states (PDOS), we use the (Kohn-Sham LDA) wavefunctions from the octopus ground state calculation and project them onto spherical harmonics according to the prescription of reference 9 used for instance in Ref. 26. Details are given in the ESI. 

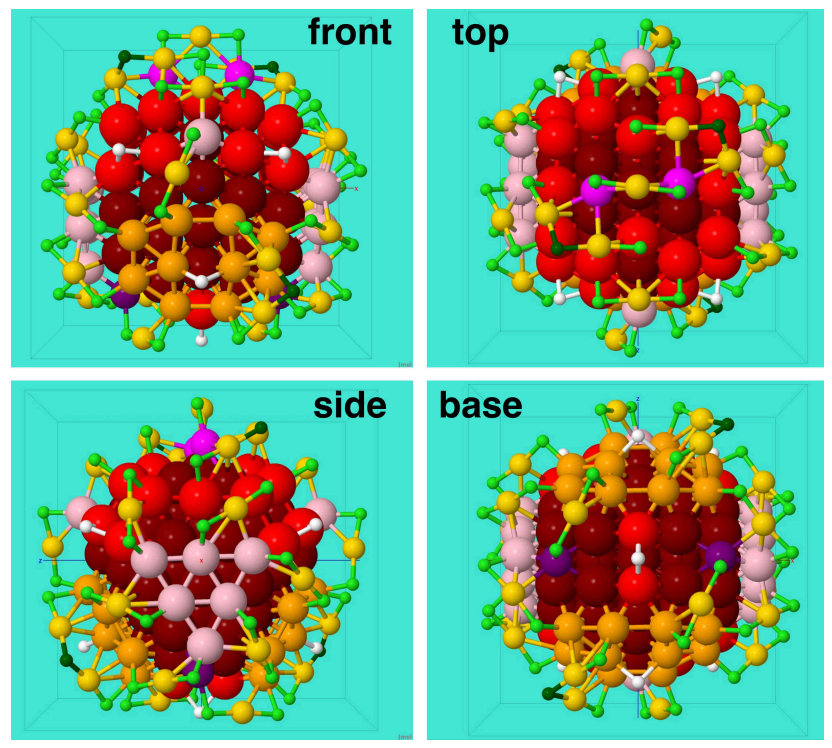

Figure 1: Geometry of the $\mathbf{A u}_{146}(\mathbf{S R})_{57}$ cluster compound with characteristic sites highlighted. Shown are the gold sites colored as described in the following, and the S atoms of the ligands. A complete figure of $\mathrm{Au}_{146}\left(\mathrm{SCH}_{3}\right)_{57}$ is shown below in Fig. 2 and in Fig. S2 of the ESI. The cluster holds $\mathrm{C}_{2}$ symmetry (the vertical y-axis corresponds to the two-fold rotation axis). Consequently, 4 of the possible 6 views suffice to present the cluster fully. "Top" and "base" views are parallel to this two-fold axis.

Color scheme for the 146 Au-sites: No Au-S bond - dark red (59); single Au-S 'surface' sites (60); red (20+2), to complete the twinned truncated-octahedron 81-site core; pink (14), magenta (2), purple (2), also on FCC lattice (99) orange $(10+10)$, completes sum to 119. (A rendition of this is seen in the ESI.) Apart from this core, there are 27 peripheral gold sites shown in yellow (small) in $\mathrm{S}-\mathrm{Au}-\mathrm{S}$ collinear bonds.

Color scheme for 57 S-sites: 7 sites bridging two core-Au-sites - white; 4 sites bridging two peripheral-Au-sites - dark green; $38+8=46$ sites bridging surface and core sites lime-green.

\section{Results and Discussion}

In Fig. 1 we show the structure of the $\mathrm{Au}_{146}(\mathrm{SR})_{57}$ cluster compound with different sites colorcoded. All gold sites and the S sites of the ligands are shown. The cluster is approximately globular and exhibits $\mathrm{C}_{2}$ symmetry. (The top view looks down along the two-fold rotation axis.) It's core consists of a twinned truncated octahedron, which is surrounded by an ordered layer of peripheral gold atoms and p-MBA ligands.

The distribution of interatomic distances, $g(d)$, is shown in Fig. 2 where we compare the values found for the experimental structure as is with the LDA-relaxed structure and, for 
comparison, with the structure relaxed using the PBE GGA functional.

Clearly, the Au-Au distances are very well reproduced with LDA, which holds not only for the nearest-neighbor distances but also for the $\mathrm{Au}-\mathrm{Au}$ distances of more distant $\mathrm{Au}$ atoms. By contrast, relaxation using the GGA functional PBE overestimates the bond lengths significantly. This is a direct confirmation of the commonly known fact that LDA yields good interatomic distances for gold, which is ex post facto a justification of using it in the present calculations. However, the preference of the LDA functional over PBE is certainly less well justified for the Au-S bonds.

Unlike the nearest-neighbor Au-Au bonds, the Au-S bond lengths show a bi-modal distribution already in the experimental coordinates, where, however, this double-peak structure is rather diffuse. These peaks get much narrower following the relaxation, indicating a slight symmetrization of the structure compared to the "noisy" data from the (average of multiple) diffraction experiments. The thin arrows indicate the average (weight) value of the peaks, using the same color as the curves. It is interesting to note that in spite of the differences between the LDA-relaxed and the experimentally determined Au-S bonds, their averages as indicated by the thicker arrows in Fig. 2 are almost identical, which serves as a further justification for the use of LDA in the relaxation.

In order to understand the origin of this bimodal distribution, we turn to the colored representation in the inset of the lower panel of Fig. 2. We color in red the S atoms which have two bonds shorter than $2.330 \AA$. (This is the value of the minimum separating the two peaks.) It turns out that these are exactly the four $\mathrm{S}$ sites that bridge two peripheral $\mathrm{Au}$ sites, as colored in dark green in Fig. 1. By contrast, the $\mathrm{S}$ atoms which have two bonds that are longer than $2.330 \AA$, rendered in blue in Fig. 2, turn out to connect two core $\mathrm{Au}$ sites (cf., white in Fig. 1). All the others (yellow) belong to "simple bridging thiolates" with one bond longer and one bond shorter than $2.330 \AA$. Inspection shows that this is similar to the situation found for the $\mathrm{Au}_{144}\left(\mathrm{SCH}_{3}\right)_{60}$ cluster where in the so-called "staple motifs" the two collinear S-Au-S bonds are shorter than the Au-S bonds connecting to the core.16 


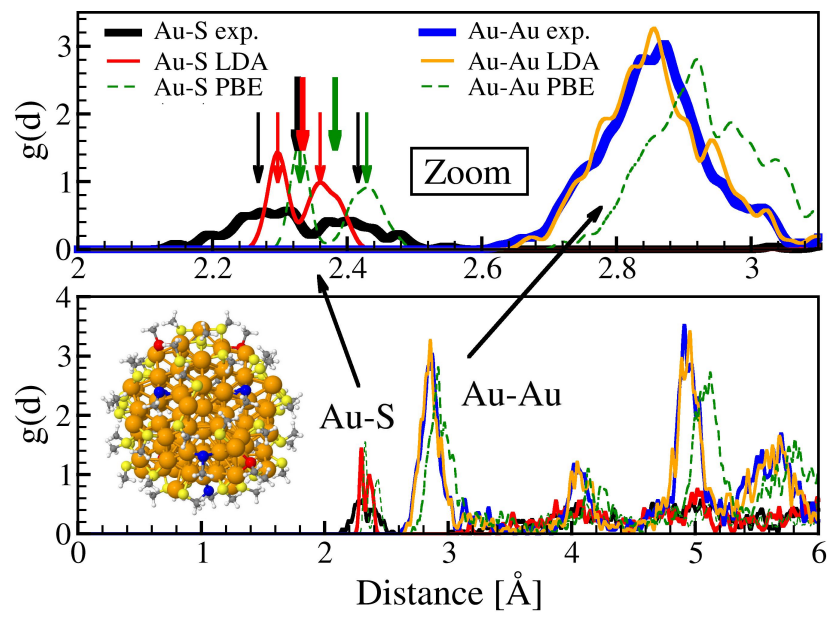

Figure 2: Interatomic distances: We show the distribution $g(d)$ of $\mathrm{Au}-\mathrm{Au}$ and $\mathrm{Au}-\mathrm{S}$ interatomic distances over a wider range (lower panel), with the upper panel showing a blow-up of the region of $\mathrm{Au}-\mathrm{Au}$ and $\mathrm{Au}-\mathrm{S}$ bonds.

We compare the unrelaxed structure from experiment as is (thick blue and black) with the structure relaxed using the LDA functional and in the 3- charge state. For comparison, the result obtained using PBE-GGA is also shown (dashed green). The thin arrows indicate the average (weight) value of the peaks, using the same color as the curves. The first peak of the experiment is centered at $2.268 \AA$, the second at $2.416 \AA$. The narrower peaks of the LDA-relaxed structure are centered at $2.297 \AA$ and $2.360 \AA$. Interestingly, the average of all $\mathrm{Au}-\mathrm{S}$ bonds (thick arrows) have almost the same distance: $2.327 \AA$ for the experiment, and $2.334 \AA$ after relaxation (LDA). By comparison, the PBE relaxation led consistently to larger values.

The inset in the lower panel shows the LDA-relaxed cluster including the methyl groups. To highlight the spatial distribution of the $\mathrm{Au}-\mathrm{S}$ bond lengths, the $\mathrm{S}$ atoms are colored blue for those with two S-Au bonds longer than $2.330 \AA$. These are the sites that connect to two core-Au sites, shown in white in Fig. 1. Colored red are those with two S-Au bonds shorter than $2.330 \AA$ (bridging two peripheral sites, dark green in Fig. 1. (The minimum separating the two peaks of the distribution of Au-S bonds is at $2.330 \AA$ for the LDA relaxed structure.) The yellow ones have both one bond longer and one shorter than $2.330 \AA$. This corresponds to the situation in $\mathrm{Au}_{144}\left(\mathrm{SCH}_{3}\right)_{60}$ where in the "staple motifs" the two collinear S-Au-S bonds are shorter than the $\mathrm{S}-\mathrm{Au}$ bonds connecting to the core. ${ }^{[16}$ All curves were obtained by convolution with a Gaussian of $\sigma=0.01 \AA$. 
In conclusion of this part, we have a rather good justification for the use of the LDA functional in the calculation, and the character of the geometric features of the ligand layer is in agreement with previously obtained results of the $\mathrm{Au}_{144}$ cluster.

In Fig. 3 we show the results of the electronic structure calculations of $\mathrm{Au}_{146}\left(\mathrm{SCH}_{3}\right)_{57}^{-3}$. In the upper panel, we show the full density of states and the individual electronic energies. As usual, one sees the clear $\mathrm{Au} 5 \mathrm{~d}$ band and additional peaks originating from the ligands. The region around the HOMO-LUMO gap is enlarged in the inset, with isosurface representations of the frontier orbitals shown. The zero of the energy axis has been set to the HOMO energy.

Comparing the electronic energies of the relaxed cluster in Fig. 3 with those shown for comparison for the as is experimental structure, one observes the effect that was to be expected from the findings of Fig. 2 which shows a symmetrization, i.e., a narrowing of the distribution of the Au-S bonds. While the eigenenergies of the unrelaxed structure are more or less equally distributed, with no discernible differences, neither degeneracies nor an appreciable gap, the gap increases significantly under the relaxation, reaching the value of $0.22 \mathrm{eV}$. Moreover, the cumulative density of states shows an inflection point, corresponding to a minimum in the density of states as it is characteristic for a SAC shell closure and indicative of "electronic stability" .9 Nonetheless, this gap remains rather small, even in view of the possibility that the LDA underestimates the gap compared to the true value. Thus no clear conclusion about any SAC character can be drawn from the gap value.

For a clearer conclusion concerning the SAC character, the angular-momentum-projected density of states is shown in the second panel of Fig. 3, again choosing the HOMO energy as origin of the energy scale. It becomes clear here that despite the small gap that might have been indicative of a SAC shell closure, the electronic states are mixtures of many different angular-momentum components. In particular, the strong P contribution (green) would not be consistent with the Aufbau rule expected in case of a SAC shell closing at 92 electrons. Upon visual inspection of the wavefunctions (Fig. S2 of the ESI) we conjecture that maybe 

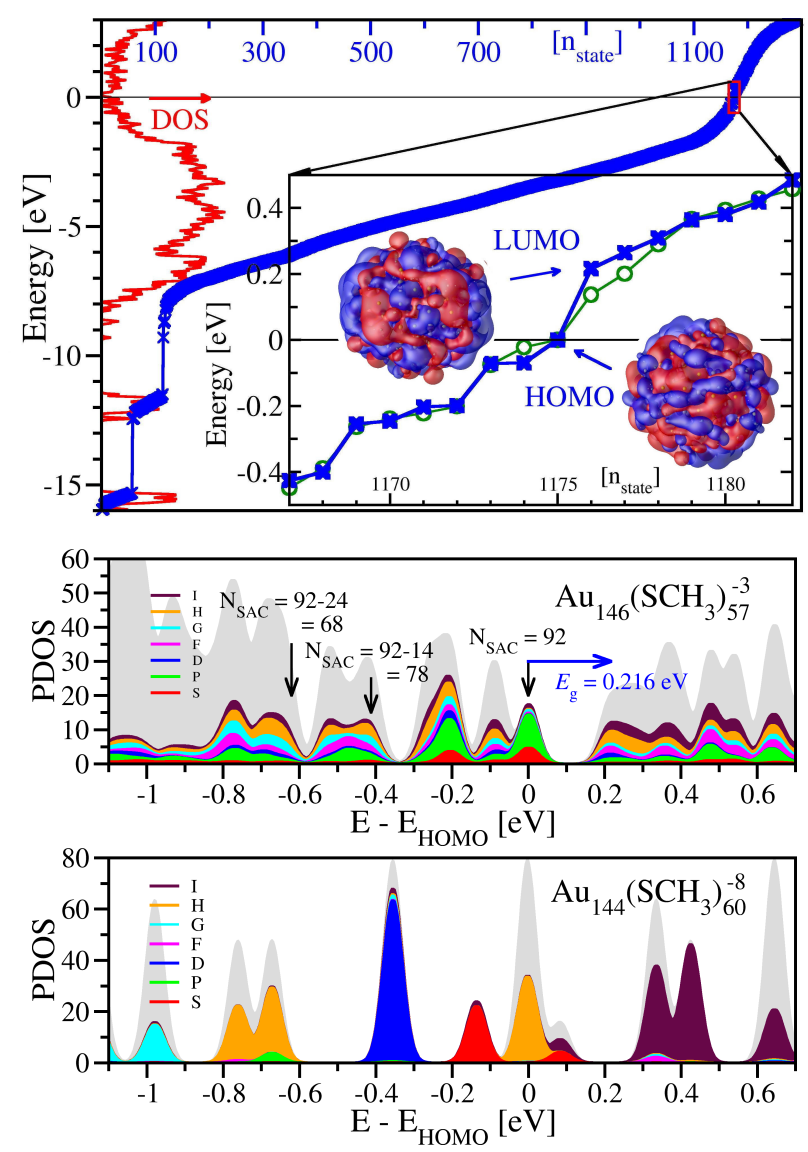

Figure 3: Electronic structure of $\mathbf{A u}_{146}\left(\mathbf{S C H}_{3}\right)_{57}^{-3}$. In the upper panel, we show the electronic density of states (red) as well as the individual energies (blue crosses) as a function of their state number. (The HOMO state corresponds to state number 1175 (2-fold degenerate for spin.)) The inset shows a zoom of the region around the gap for the relaxed structure (blue crosses) and the as-is experimental structure (green circles). In addition, the wavefunctions of the nondegenerate HOMO and LUMO states are shown (iso value of $0.001 \AA^{-3}$.)

Below, we show the angular-momentum-projected DOS of $\mathrm{Au}_{146}\left(\mathrm{SCH}_{3}\right)_{57}^{-3}$. Clearly, in spite of the small gap at the potential shell closing at $92 \mathrm{SAC}$ electrons, the electronic states do not show any clear SAC character, as they are by and large mixtures of different angular-momentum components. This contrasts strongly with the case of the fully symmetric $\mathrm{Au}_{144}\left(\mathrm{SCH}_{3}\right)_{60}^{-8}$ cluster, shown for comparison, the states of which exhibit clear SAC character. (The high charge state -8 was chosen to obtain a number of SAC electrons of 92; the structure was from the neutral calculation.) Note that the result is slightly modified compared to the projected DOS of Ref. 26 because the fully symmetrized structure of Ref. 39 has been used in the present calculation. The angular-momentum components are as indicated in the legend, while the grey area reports the full, integrated DOS. Note that the DOS of $\mathrm{Au}_{144}\left(\mathrm{SCH}_{3}\right)_{60}$ is more strongly peaked than that of $\mathrm{Au}_{146}\left(\mathrm{SCH}_{3}\right)_{57}^{-3}$ due to the much higher symmetry-related degeneracy.

All energies are Kohn-Sham energies from the LDA calculation; the DOS is convoluted with a gaussian of $\sigma=0.025 \mathrm{eV}$. The zero of all energy axes was set to the HOMO energy. 
a modified ellipsoidal SAC model would be better adapted to describe the cluster. In Fig. S2 of the ESI we show the frontier orbitals in different representations for illustration, along with the geometry of the $\mathrm{R}=\mathrm{CH}_{3}$ model used in the calculation.

To further confirm these findings, we compare with the case of the fully $\mathrm{I}_{h}$-symmetric $\mathrm{Au}_{144}\left(\mathrm{SCH}_{3}\right)_{60}$ cluster $\underline{1516139}$ in the lower panel of Fig. 3. (The high charge 8- was chosen to obtain a SAC electron count of 92 , corresponding to a possible shell closure. The structure was that of the neutral calculation. $\frac{39}{3}$ ) The difference between the two systems is striking: most of the $\mathrm{Au}_{144}$ states exhibit rather pure SAC character with distinct angular-momenta, even though the condition of a shell closing at $92 \mathrm{SAC}$ electrons is not fulfilled. (The HOMOLUMO gap is actually very small; the SAC gap is at $\mathrm{HOMO}+1$.) Second, the DOS is more peaked in this case, indicating a higher degree of degeneracy due to the high symmetry.

In Fig. 4, we report the calculated absorption spectra for light polarized along the Cartesian directions alongside the absorption averaged over all directions that would be measured in a solution-phase experiment where the orientation of the clusters is random. As to be expected in view of the symmetry, the absorption of the cluster is not isotropic. However, the differences between the directions are relatively small.

The average absorption of $\mathrm{Au}_{146}\left(\mathrm{SCH}_{3}\right)_{57}$ shows comparably little structure, in particular by comparison with the spectrum of the $\mathrm{Au}_{144}\left(\mathrm{SCH}_{3}\right)_{60}$ cluster which is isotropic due to the high symmetry, and which has been reported before. $\frac{15 \mid 16}{16}$ The reasons for this remarkable difference are two-fold: first, the average over the different directions has a smoothing effect. Second, the $\mathrm{I}_{h}$ symmetry of $\mathrm{Au}_{144}\left(\mathrm{SCH}_{3}\right)_{60}$ leads to a high degree of degeneracy in the electronic states, which is in turn visible in the spectra. By comparison, as mentioned above, $\mathrm{Au}_{146}\left(\mathrm{SCH}_{3}\right)_{57}$ shows little symmetry-related degeneracy; its DOS is less peaked than that of the icosahedral $\mathrm{Au}_{144}$ cluster, which translates into smoother spectra.

These findings mean that the optical spectra measured in solution will not easily find individual spectral structures that calculations could be compared with. We hope that these 


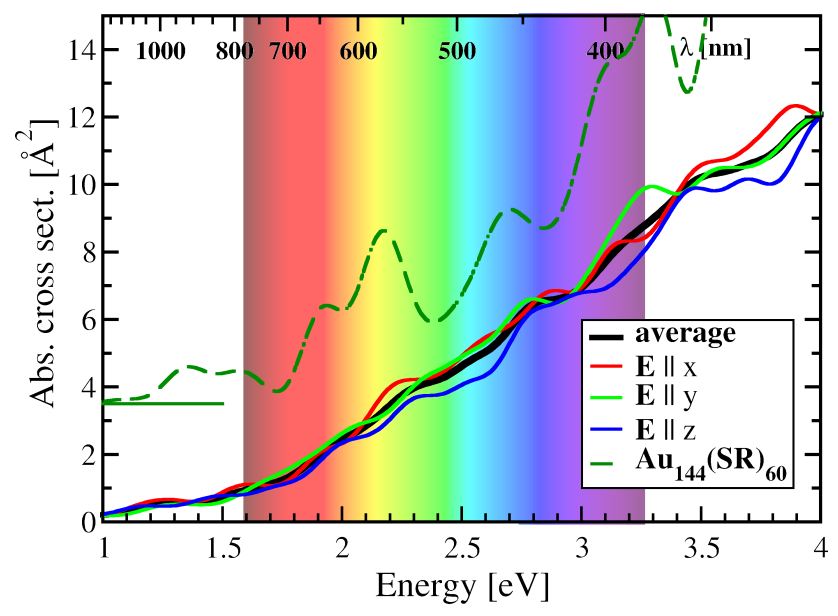

Figure 4: Optical absorption spectrum of $\mathbf{A u}_{146}\left(\mathbf{S C H}_{3}\right)_{57}^{-3}$. The black line shows the average (trace of the full absorption cross-section tensor), while the red, green, and blue lines show the absorption for light polarized along $\mathrm{x}, \mathrm{y}$, and $\mathrm{z}$, respectively. Clearly, the system is not isotropic. However, its spectra show comparably little structure compared with the spectrum of the $\mathrm{Au}_{144}\left(\mathrm{SCH}_{3}\right)_{60}$ cluster which is shown for comparison (Green dashed line, shifted for visibility). The latter system is isotropic due to its high symmetry $\left(\mathrm{I}_{h}\right)$. Moreover, this high symmetry leads to higher degeneracy of electronic states, which in turn results in clear, discrete structures in the spectra. The electronic states of $\mathrm{Au}_{146}\left(\mathrm{SCH}_{3}\right)_{57}^{-3}$, by contrast, show little degeneracy (cf., Fig. 3) and the resulting optical response spectrum is rather smooth. (The spectra have been calculated using the PBE functional in order to be consistent with earlier calculations; a comparison with LDA spectra is shown in the ESI.) 
results motivate the measurement of optical absorption on pure $\mathrm{Au}_{146}(\mathrm{pMBA})_{57}$ crystals in order to obtain spectra for well-defined directions.

\section{Conclusions}

We have studied the electronic structure and the optical properties of the novel monolayerprotected gold cluster $\mathrm{Au}_{146}(\mathrm{pMBA})_{57}$, the structure of which has been recently determined by Vergara et al..$^{1}$ It is the largest aqueous gold cluster ever structurally determined and likewise the smallest cluster with a stacking fault.

The density-functional theory calculations were carried out for the charge state 3- (three additional electrons), motivated by the fact that this charge results in a number of $92 \mathrm{SAC}$ electrons for which a major shell closing is expected in case the cluster conforms with the SAC model. The ligand rest groups were replaced by methyl groups, $\mathrm{CH}_{3}$.

Relaxation with the LDA functional resulted in close agreement with the experimental $\mathrm{Au}-\mathrm{Au}$ distances. The relaxed structure kept all the connectivity of the original coordinates from the experiment, whereas much of the irregularity of the interatomic distances was removed, enhancing the $\mathrm{C}_{2}$ symmetry feature.

Study of the angular-momentum-projected density of states allows us to draw the following central conclusion of the present study: while there is a small gap of the order of $0.2 \mathrm{eV}$, the electronic states do not even approximately exhibit SAC character. The strong P-type component of the states close to the HOMO is likewise inconsistant with the Aufbauprinzip which would expect even $\mathrm{L}$ values to dominate. Thus one cannot attribute the stability of the $\mathrm{Au}_{146}(\mathrm{SR})_{57}$ compound to a superatomic shell closing, despite the proximity of the magic number of $92 \mathrm{SAC}$ electrons.

Finally, we have shown that relatively weak individual structures are found in the absorption spectra. We hope that these results can motivate experimental measurements of the absorption spectra on crystallized samples. 


\section{Acknowledgments}

Enlightening discussions with Andres Saul and Ignacio Garzón are gratefully acknowledged. X.L.L. acknowledge previous funding from NSF-DMR-1103730 and NSF-PREM DMR-0934218. RLW acknowledges the support of the Welch Foundation under grant AX-1857. HCW acknowledges support from the French National Research Agency (Agence Nationale de Recherche, ANR) in the frame of the project "FIT SPRINGS", ANR-14-CE08-0009. This work has used HPC resources from GENCI-IDRIS (Grant 096829) and from the Laboratory of Computational Nanotechnology and UTSA Research Computing Support Group through the HPC clusters Antares3 and SHAMU, respectively, as well as the Texas Advanced Computing Center (TACC) at the University of Texas at Austin.

\section{References}

(1) Sandra Vergara, Dylan Lukes, Ulises Santiago, German Plascencia-Villa, Simon C. Weiss, Michael W. Martynowycz, M. Jason de la Cruz, David M. Black, Marcos M. Alvarez, Xochitl Lopez-Lozano, Christopher O. Barnes, Hans-Christian Weissker, Robert L. Whetten, Tamir Gonen, Guillermo Calero, submitted to the Science journal, available at arXiv:1706.07902.

(2) Hainfeld, J. F.; Liu, W.; Halsey, C. M.; Freimuth, P.; Powell, R. D. Ni-NTA-Gold Clusters Target His-Tagged Proteins. Journal of Structural Biology 1999, 127, 185 198.

(3) Ackerson, C. J.; Jadzinsky, P. D.; Sexton, J. Z.; Bushnell, D. A.; Kornberg, R. D. Synthesis and Bioconjugation of 2 and 3 nm-Diameter Gold Nanoparticles. Bioconjugate Chemistry 2010, 21, 214-218, PMID: 20099843.

(4) Ackerson, C. J.; Powell, R. D.; Hainfeld, J. F. In Cryo-EM Part A Sample Preparation 
and Data Collection; Jensen, G. J., Ed.; Methods in Enzymology; Academic Press, 2010; Vol. 481; pp 195 - 230.

(5) Bowman, M.-C.; Ballard, T. E.; Ackerson, C. J.; Feldheim, D. L.; Margolis, D. M.; Melander, C. Inhibition of HIV Fusion with Multivalent Gold Nanoparticles. Journal of the American Chemical Society 2008, 130, 6896-6897.

(6) Bresee, J.; Maier, K. E.; Boncella, A. E.; Melander, C.; Feldheim, D. L. Growth Inhibition of Staphylococcus aureus by Mixed Monolayer Gold Nanoparticles. Small 2011, 7, 2027-2031.

(7) Khanna, S. N.; Jena, P. Assembling crystals from clusters. Phys. Rev. Lett. 1992, 69, $1664-1667$.

(8) Ekardt, W. Work function of small metal particles: Self-consistent spherical jelliumbackground model. Phys. Rev. B 1984, 29, 1558-1564.

(9) Walter, M.; Akola, J.; Lopez-Acevedo, O.; Jadzinsky, P. D.; Calero, G.; Ackerson, C. J.; Whetten, R. L.; Grönbeck, H.; Häkkinen, H. A unified view of ligand-protected gold clusters as superatom complexes. Proceedings of the National Academy of Sciences 2008, 105, 9157-9162.

(10) Wyrwas, R. B.; Alvarez, M. M.; Khoury, J. T.; Price, R. C.; Schaaff, T. G.; Whetten, R. L. The colours of nanometric gold. The European Physical Journal D 2007, 43, 91-95.

(11) Yoon, B.; Koskinen, P.; Huber, B.; Kostko, O.; von Issendorff, B.; Häkkinen, H.; Moseler, M.; Landman, U. Size-Dependent Structural Evolution and Chemical Reactivity of Gold Clusters. ChemPhysChem 2007, 8, 157-161.

(12) Jin, R.; Zeng, C.; Zhou, M.; Chen, Y. Atomically Precise Colloidal Metal Nanoclusters 
and Nanoparticles: Fundamentals and Opportunities. Chemical Reviews 2016, 116, $10346-10413$.

(13) Malola, S.; Lehtovaara, L.; Enkovaara, J.; Häkkinen, H. Birth of the Localized Surface Plasmon Resonance in Monolayer-Protected Gold Nanoclusters. ACS Nano 2013, 7, $10263-10270$.

(14) Qian, H.; Zhu, Y.; Jin, R. Atomically precise gold nanocrystal molecules with surface plasmon resonance. Proceedings of the National Academy of Sciences 2012,

(15) Weissker, H.-C.; Escobar, H. B.; Thanthirige, V. D.; Kwak, K.; Lee, D.; Ramakrishna, G.; Whetten, R.; ; López-Lozano, X. Information on quantum states pervades the visible spectrum of the ubiquitous Au144 gold nanocluster. Nature Communications $\mathbf{2 0 1 4}, 5,3785$.

(16) Weissker, H.-C.; Lopez-Acevedo, O.; Whetten, R. L.; Lopez-Lozano, X. Optical Spectra of the Special Au144 Gold-Cluster Compounds: Sensitivity to Structure \& Symmetry. The Journal of Physical Chemistry $C$ 0, 0, null.

(17) Kumara, C.; Dass, A. $(\mathrm{AuAg})_{144}(\mathrm{SR})_{60}$ alloy nanomolecules. Nanoscale 2011, 3, 30643067.

(18) Malola, S.; Lehtovaara, L.; Häkkinen, H. TDDFT Analysis of Optical Properties of Thiol Monolayer-Protected Gold and Intermetallic Silver-Gold Au144(SR)60 and Au84Ag60(SR)60 Clusters. The Journal of Physical Chemistry C 2014, 118, 2000220008.

(19) Barcaro, G.; Sementa, L.; Fortunelli, A.; Stener, M. Comment on "(Au-Ag)144(SR)60 alloy nanomolecules" by C. Kumara and A. Dass, Nanoscale, 2011, 3, 3064. Nanoscale $2014,-$. 
(20) López-Lozano, X.; Mottet, C.; Weissker, H.-C. Effect of Alloying on the Optical Properties of Ag/Au Nanoparticles. The Journal of Physical Chemistry C 2013, 117, 30623068.

(21) Dharmaratne, A. C.; Dass, A. $\mathrm{Au}_{144-x} \mathrm{Cu}_{x}\left(\mathrm{SC}_{6} \mathrm{H}_{13}\right)_{60}$ nanomolecules: effect of $\mathrm{Cu}$ incorporation on composition and plasmon-like peak emergence in optical spectra. Chem. Commun. 2014, 50, 1722-1724.

(22) Bhattarai, N.; Black, D. M.; Boppidi, S.; Khanal, S.; Bahena, D.; Tlahuice-Flores, A.; Bach, S. B. H.; Whetten, R. L.; Jose-Yacaman, M. ESI-MS Identification of Abundant Copper-Gold Clusters Exhibiting High Plasmonic Character. The Journal of Physical Chemistry C 2015, 119, 10935-10942.

(23) Sinha-Roy, R.; López-Lozano, X.; Whetten, R. L.; García-González, P.; Weissker, H.C. In Search of the Quantum-Electronic Origin of Color Change: Elucidation of the Subtle Effects of Alloying with Copper on $\approx 1.8 \mathrm{~nm}$ Gold Nanoclusters. The Journal of Physical Chemistry C 2017, 121, 5753-5760.

(24) Negishi, Y.; Nakazaki, T.; Malola, S.; Takano, S.; Niihori, Y.; Kurashige, W.; Yamazoe, S.; Tsukuda, T.; Häkkinen, H. A Critical Size for Emergence of Nonbulk Electronic and Geometric Structures in Dodecanethiolate-Protected Au Clusters. Journal of the American Chemical Society 2015, 137, 1206-1212, PMID: 25549276.

(25) Jensen, K. M. Ø.; Juhas, P.; Tofanelli, M. A.; Heinecke, C. L.; Vaughan, G.; Ackerson, C. J.; Billinge, S. J. L. Polymorphism in magic-sized Au144(SR)60 clusters. Nature Communications 2016, 7, 11859.

(26) Lopez-Acevedo, O.; Akola, J.; Whetten, R. L.; Grönbeck, H.; Häkkinen, H. Structure and Bonding in the Ubiquitous Icosahedral Metallic Gold Cluster $\operatorname{Au}_{144}(\mathrm{SR})_{60}$. The Journal of Physical Chemistry C 2009, 113, 5035-5038. 
(27) Kresse, G.; Hafner, J. Ab initio molecular dynamics for liquid metals. Phys. Rev. B 1993, $47,558$.

(28) Kresse, G.; Furthmüller, J. Efficiency of ab-initio total energy calculations for metals and semiconductors using a plane-wave basis set. Comput. Mat. Sci. 1996, 6, 15.

(29) Kresse, G.; Joubert, D. From ultrasoft pseudopotentials to the projector augmentedwave method. Phys. Rev. B 1999, 59, 1758.

(30) Perdew, J. P.; Burke, K.; Ernzerhof, M. Generalized Gradient Approximation made simple. Phys. Rev. Lett. 1996, 77, 3865.

(31) Marques, M. A. L.; Castro, A.; Bertsch, G. F.; Rubio, A. Comp. Phys. Comm. 2003, $151,60$.

(32) Castro, A.; Marques, M. A. L.; Appel, H.; Oliveira, M.; Rozzi, C.; Andrade, X.; Lorenzen, F.; Gross, E. K. U.; Rubio, A. Phys. Stat. Sol. (b) 2006, 243, 2465.

(33) Yabana, K.; Bertsch, G. F. Time-dependent local-density approximation in real time. Phys. Rev. B 1996, 54, 4484-4487.

(34) Troullier, N.; Martins, J. L. Efficient pseudopotentials for plane-wave calculations. Phys. Rev. B 1991, 43, 1993-2006.

(35) Castro, A.; Marques, M. A. L.; Rubio, A. Propagators for the time-dependent KohnSham equations. The Journal of Chemical Physics 2004, 121, 3425-3433.

(36) Casida, M. E. Recent Advances in Density Functional Methods, Part I. 1995; p 155.

(37) Casida, M. E. Recent Developments and Applications of Modern Density Functional Theory. 1996; p 391. 
(38) Lopez-Lozano, X.; Barron, H.; Mottet, C.; Weissker, H.-C. Aspect-ratio- and sizedependent emergence of the surface-plasmon resonance in gold nanorods - an ab initio TDDFT study. Phys. Chem. Chem. Phys. 2014, 16, 1820-1823.

(39) Bahena, D.; Bhattarai, N.; Santiago, U.; Tlahuice, A.; Ponce, A.; Bach, S. B. H.; Yoon, B.; Whetten, R. L.; Landman, U.; Jose-Yacaman, M. STEM Electron Diffraction and High-Resolution Images Used in the Determination of the Crystal Structure of the $\mathrm{Au}_{144}(\mathrm{SR})_{60}$ Cluster. The Journal of Physical Chemistry Letters 2013, 4, 975-981. 